\title{
Differential expression of protein disulfide-isomerase $A 3$ isoforms, PDIA3 and PDIA3N, in human prostate cancer cell lines representing different stages of prostate cancer
}

\author{
Maria Araceli Diaz Cruz $^{1}$ [D $\cdot$ Sandra Karlsson ${ }^{2} \cdot$ Ferenc Szekeres $^{3} \cdot$ Maria Faresjö $^{2} \cdot$ Dan Lund $^{2} \cdot$ Dennis Larsson $^{4}$
}

Received: 13 October 2020 / Accepted: 11 March 2021 / Published online: 24 March 2021

(C) The Author(s) 2021

\begin{abstract}
Prostate cancer (PCa) is a highly heterogeneous and unpredictable progressive disease. Sensitivity of PCa cells to androgens play a central role in tumor aggressiveness but biomarkers with high sensitivity and specificity that follow the progression of the disease has not yet been verified. The vitamin D endocrine system and its receptors, the Vitamin D Receptor (VDR) and the Protein Disulfide-Isomerase A3 (PDIA3), are related to anti-tumoral effects as well as carcinogenesis and have therefore been suggested as potential candidates for the prevention and therapy of several cancer forms, including PCa. In this study, we evaluated the mRNA expression of VDR and PDIA3 involved in vitamin D signaling in cell lines representing different stages of PCa (PNT2, P4E6, LNCaP, DU145 and PC3). This study further aimed to evaluate vitamin D receptors and their isoforms as potential markers for clinical diagnosis of PCa. A novel transcript isoform of PDIA3 (PDIA3N) was identified and found to be expressed in all PCa cell lines analyzed. Androgen-independent cell lines showed a higher mRNA expression ratio between PDIA3N/PDIA3 contrary to androgen-dependent cell lines that showed a lower mRNA expression ratio between PDIA3N/PDIA3. The structure of PDIA3N differed from PDIA3. PDIA3N was found to be a N-truncated isoform of PDIA3 and differences in protein structure suggests an altered protein function i.e. cell location, thioredoxin activity and affinity for $1,25(\mathrm{OH})_{2} \mathrm{D}_{3}$. Collectively, PDIA3 transcript isoforms, the ratio between PDIA3N/PDIA3 and especially PDIA3N, are proposed as candidate markers for future studies with different stages of PCa progression.
\end{abstract}

Keywords Prostate cancer $\cdot$ Vitamin D $\cdot$ PDIA3 $\cdot$ PDIA3N $\cdot$ VDR $\cdot$ Androgen dependency

\section{Introduction}

Prostate cancer $(\mathrm{PCa})$ is one of the most common cancer types worldwide and the second leading cause of death among men in the United States and Europe $[1,2]$. The disease is heterogeneous and unpredictable since prostate

Maria Araceli Diaz Cruz

Maria-Araceli.Cruz@ju.se

1 Research School of Health and Welfare, School of Health and Welfare, University of Jönköping, Jönköping, Sweden

2 Department of Natural Science and Biomedicine, School of Health and Welfare, University of Jönköping, Jönköping, Sweden

3 Department of Biomedicine, School of Health Sciences, University of Skövde, Skövde, Sweden

4 Sahlgrenska University Hospital, Gothia Forum - for Clinical Research, Gothenburg, Sweden cancer cells easily can pass from indolent to rapidly progressive and may lead to fatality [3].

A major clinical challenge in PCa screening is the lack of diagnostic tests that distinguish between benign and aggressive tumors [1, 4]. Standard approaches used are tissue pathology and Gleason score, imaging and prostate specific antigen (PSA) serum levels $[1-3,5]$. Analyses of tissue pathology and Gleason score classifications are more reliable but require invasive techniques to extract biopsies from the tumor. Imaging is not used if tumor growth is not expected and usually miss small tumors. PSA screening is the most effective non-invasive method used for more than 20 years. However, PSA screening has low sensitivity and marginal specificity resulting in high number of false positives associated with other uropathies [4]. Thus, there is a need of finding candidate biomarkers with both high sensitivity and specificity to follow PCa progression.

New gene transcript isoforms are emerging as suitable candidates to represent disease progression since high 
concentrations of specific protein isoforms may be associated with specific tumor stages or level of disease progression $[6,7]$. An example is the discovery of an alternative splicing isoform signature associated with overall survival for hepatocellular carcinoma [8]. Also, the isoform switches are highly predictive for cancer survival and aggressiveness, such as the DNA excision repair 1 (ERCC1) gene resulting in a protein lacking the HHH domain associated with lower cancer survival rates [9].

Vitamin D and its metabolites have been suggested as potential candidates for the prevention and therapy of several cancer forms, including PCa [10,11]. Numerous reports demonstrate that vitamin $\mathrm{D}$, through 1,25-dihydroxyvitamin $\mathrm{D}_{3}\left(1,25(\mathrm{OH})_{2} \mathrm{D}_{3}\right)$, exerts antitumor effects. $1,25(\mathrm{OH})_{2} \mathrm{D}_{3}$ has been demonstrated to stimulate differentiation, increase apoptosis and inhibit proliferation, invasiveness and metastasis of cancer cells [11, 12]. Two receptors are known to be involved in the vitamin D signaling pathway and its mediated effects. The nuclear vitamin D receptor (VDR) and the protein disulfide isomerase family A, member 3 (PDIA3) [13-15]. VDR is localized in the cytosol and becomes activated upon binding with $1,25(\mathrm{OH})_{2} \mathrm{D}_{3}$, leading to a heterodimerization with the RXR receptor. This complex migrates to the nucleus where it modulates gene transcription after binding with the vitamin D response element (VDRE) [16, 17]. PDIA3 is a chaperone protein localized mainly in the endoplasmic reticulum, suggested to be responsible for "rapid actions" of $1,25(\mathrm{OH})_{2} \mathrm{D}_{3}$ initiated at the plasma membrane $[13,14]$. These actions include regulation of intracellular, extranuclear pathways and signaling cascades, such as the activation of the protein kinase $\mathrm{C}$ (PKC) pathway and the calcium transport [13]. The role of PDIA3 in cancer regulation remains controversial since some studies suggest that it is responsible for the activation of proapoptotic pathways $[18,19]$ whereas others suggest association with cancer proliferation, inhibition of apoptosis and poor prognosis [20, 21]. In both normal and cancer prostate tissues, VDR is found to be lower both at level of transcription and translation compared to PDIA3 [22].

We evaluated mRNA expression of VDR and PDIA3 involved in vitamin $\mathrm{D}$ signaling in cell lines representing different stages of PCa (PNT2, P4E6, LNCaP, DU145 and PC3). Further, we aimed to evaluate vitamin D receptors and their isoforms as potential markers for clinical diagnosis of PCa.

\section{Materials and methods}

\section{In vitro cell culture}

Following commercial prostate cells, representing different stages of prostate cancer, were used in the present study
(Supplementary File 1); PNT2, P4E6 and LNCaP (Sigma Aldrich, St. Louis, MO, USA), DU145 and PC3 (ATCC, Manassas, VA, USA). PNT2 and LNCaP cells were cultured in RPMI-1640 supplemented with 10\% FBS and 1\% PEST (Sigma Aldrich), whereas P4E6 cells were maintained in Stemline Keratinocyte Medium II with Stemline Keratinocyte Growth Supplement, 2 mM Glutamine and 2\% of FBS (Sigma Aldrich), DU145 cells were cultured in EMEM (Sigma Aldrich) supplemented with 10\% FBS and 1\% PEST and PC 3 cells were cultured in DMEM (Sigma Aldrich) containing 10\% FBS, 5\% of pyruvate sodium (Sigma Aldrich) and $1 \%$ PEST.

\section{RNA extraction and reverse transcription (RT-PCR)}

Total RNA was extracted from approximately $1 \times 10^{6}$ cells, using RNeasy Mini Kit including genomic DNA digestion with DNase (Qiagen, Hilden, DE). High quality RNA $(\mathrm{A} 260 / 280=1.9-2.0, \mathrm{~A} 260 / 230=1.5-1.8$, RNA integrity number $\geq 8$ ) from the respective cell line was sent for NGS analysis to The National Genomics Infrastructure in The Science Life Lab in Stockholm, Sweden (NGI, SciLifeLab).

For digital droplet PCR preparation, RNA (up to $2 \mu \mathrm{g}$ ) extracted from PNT2, DU145, PC3 and LNCaP cells was reversed transcribed to cDNA according to the manufacturer's protocol for the High Capacity cDNA Reverse Transcription Kit (Applied Biosystems, Foster City, CA, USA).

\section{New generation sequencing (NGS)}

A total of 20 ug of RNA from PNT2, P4E6, DU145, PC3 and $\mathrm{LNCaP}$ cells were sequenced with Illumina RNAseq HiSeq 2500 High Output v4, $2 \times 125$ bp in one lane giving $>37.6 \mathrm{M}$ read pairs/sample.

Software TopHat/2.0.4 was used for mapping reads to the Human genome assembly, build GRCh37 (hg19). Quantification of normalized expression genes and their different transcripts isoforms were obtained as FPKM (Fragments per Kilobase of Exon per Million Fragments Mapped) generated by cufflinks/2.1.1.

\section{Droplet digital ${ }^{\mathrm{TM}} \mathrm{PCR}$ (ddPCR)}

Droplet digital polymerase chain reaction was performed according to the manufacturer's protocol (ddPCR; QX200, Bio-Rad, Hercules, CA, USA). The ddPCR assays, consisting of two primers and TaqMan hydrolysis probe for each target, were designed using Primer3 Plus web interface [23] (Supplementary File 2). A total of 225 cDNA samples (5 cell lines, 3 biological samples $\times 3$ different passage numbers and 5 technical replicates for each sample) were analyzed to quantify the amount of PDIA3-Normal and PDIA3-Novel 
target. The amount of cDNA for each reaction was 10,000 copies corresponding to $33 \mathrm{ng}$ of DNA. Each sample was partitioned into 10,000-18,000 droplets and transferred to a 96-well-plate for subsequent PCR amplification.

After amplification, droplets were read on a QX200 droplet reader (Bio-Rad) and analyzed with QuantaSoft software V1.7.4 (Bio-Rad). Amplitude limit was set manually to 2000 for PDIA3 and 4000 for PDIA3N. The Poisson-corrected determination of mRNA expression or template concentration (copies/ $\mu \mathrm{l}$ ) and the ratio of mRNA expression between the isoforms (PDIA3-Novel/PDIA3-Normal) were calculated using QuantaSoft ${ }^{\mathrm{TM}}$ Analysis Pro Software (v1.0.596, Bio-Rad). As validation, the same experiment was repeated using 45 randomly selected samples.

\section{Statistical analysis}

Nonparametric tests were used to determine differences in mRNA expression between PCa cell lines assuming that the data did not follow a normal (Gaussian) distribution (Kolmogorov-Smirnov test and D'Agostino \& Pearson test, $\mathrm{P}$ value $<0.0001)$. Differences between ratios of mRNA expression among PCa cell lines were assessed by non-parametric Kruskal-Wallis multiple comparison test. Pairwise comparison between PCa cell lines were assessed by Mann-Whitney $U$-test. Pairwise comparisons in the same $\mathrm{PCa}$ cell line were assessed with Wilcoxon signed rank test. All statistical tests were performed with GraphPad Prism version 8.3 for Windows (GraphPad Software, La Jolla, CA, USA). Levels of significance were set to $*$ : $\mathrm{P}<0.05$, **: $\mathrm{P}<0.01, * * *: \mathrm{P}<0.001$ and $* * * *: \mathrm{P}<0.0001$.

\section{Functional analysis}

Protein sequences were retrieved from UniprotKB (P30101) for PDIA3 and from UniParc (UPI000066D935) for PDIA3N [24]. Both protein sequences were analyzed by I-TASSER (Iterative Threading ASSEmbly Refinement server) to generating the protein structure model [25]. Differences in protein sequences were highlighted with UCSF Chimera 1.0 [26]. A prediction of damage or pathogenicity of the 56 variations (amino acid substitutions and deletions) that PDIA3N present in comparison with PDIA3 was carried out with PolyPhen-2 [27] and PROVEAN (Protein Variation Effect Analyzer) v1.1 [28]. A prediction of subcellular localization of PDIA3 and PDIA3N was performed by DeepLoc-1.0 [29].

To address differences in $1,25(\mathrm{OH})_{2} \mathrm{D}_{3}$ binding with PDIA3 and PDIA3N, an automated docking of ligand to macromolecular receptor, without allowing flexible conformation, was performed with Autodock 4.0 software and analyzed with Autodock tools (ADT) [30]. Input coordinates of $1,25(\mathrm{OH})_{2} \mathrm{D}_{3}$ were extracted from the crystal structure of
VDR complexed bound to $1,25(\mathrm{OH})_{2} \mathrm{D}_{3}$ [31]. Grid spacing was set to $0.375 \AA$, number of energy evaluations was set to $2.5 \times 10^{6}$ and 150 as population size for PDIA3 and VDR and $5 \times 10^{6}$ and 500 for PDIA3N, respectively. Putative binding sites in PDIA3 and PDIA3N were selected by searching for free binding energies similar to the obtained in previous experiments with VDR and $1,25(\mathrm{OH})_{2} \mathrm{D}_{3}[15,32]$.

\section{Results}

Five prostate cell lines, PNT2, P4E6, DU145, PC3 and LNCaP, were analyzed by NGS and all these cells lines showed low mRNA expression of VDR and RXRA (0-7.4 FPKMs). A novel transcript isoform of PDIA3 (PDIA3N, ENST00000538521.1, GRCh37.p13 Ensembl 2018) [33] was also detected in all five prostate cell lines (Fig. 1A). PDIA3N was found to be 1628 bp shorter than PDIA3 (ENST00000300289, GRCh38.p12 Ensembl 2018 [33] and contained a different nucleotide sequence fragment of $178 \mathrm{bp}$, which included an alternative translation initiation site (TIS; secondary starting codon "ATG", Fig. 1a). PDIA3N had higher mRNA expression compared to PDIA3 in prostate cells with normalized expression values of 8.5-14.5 and 44.6-69.3 FPKM, respectively (Fig. 1b). Expression of PDIA3N was correlated to tumor stage, being more expressed in metastatic cell lines (DU145, PC3 and $\mathrm{LNCaP}$ ) compared to cell lines derived from normal prostate tissue (PNT2) and the cell line derived from an early stage of androgen negative prostate cancer (P4E6). The highest expression of PDIA3N (69.3 FPKM) was obtained from the metastatic androgen dependent cell line LNCaP (Fig. 1b).

Dependent on the findings in the NGS analyses of a novel isoform of PDIA3, mRNA expression of PDIA3 and PDIA3N were further evaluated by ddPCR in PNT2, P4E6, DU145, PC3 and LNCaP cells. Among AR positive cell lines, LNCaP showed a higher mRNA expression of PDIA3N compared to PDIA3 ( $<<0.01)$, whereas PNT2 showed a similar trend $(\mathrm{p}=0.063)$. Contrary to AR positive cell lines, AR negative cell lines P4E6 $(\mathrm{p}<0.05)$, DU145 (0.063) and PC3 (0.69) showed a lower or an equally high mRNA expression of PDIA3N compared to PDIA3 (Fig. 2a). AR negative cell lines (P4E6, DU145 and PC3) showed lower PDIA3N/PDIA3 ratio compared to AR positive cell lines (PNT2 and LNCaP) $(\mathrm{p}<0.0001)$. PDIA3N/PDIA3 was higher in the metastatic cell line LNCaP compared to the normal epithelial cell line PNT2 ( $p<0.0001$, Fig. 2b). PDIA3N/PDIA3 was lower in P4E6, DU145 and PC3 compared to the normal epithelial cell line PNT2 (p $<0.001$, Fig. 2b). Expression of PDIA3 and PDIA3N was higher in LNCaP cells compared to PNT2 cells $(\mathrm{p}<0.001$ and $\mathrm{p}<0.0001$ respectively; Fig. 3). 
a

ENST00000300289 (PDIA3 Normal) AGACGCGCGAGCGCAAGCAGCGGGTTAGTGGTCGCGCGCCCG
ACCTCCGCAGTCCCAGCCGAGCCGCGACCCTTCCGGCCGTCCC
CACCCCACCTCGCCGCCATGCGCCTCCGCCGCCTAGCGC
TGTTCCCGGGTGTGGCGCTGCTTCTTGCCGCGGCCCG
CCTCGCCGCTGCCTCCGACGTGCTAGAACTCACGGAC
GACAACTTCGAGAGTCGCATCTCCGACACGGGCTCTG
GGGGCCTCATGCTCGTCGAGTTCTTCGCCCCCTGG...

Exons: 13, Coding exons: 13, Transcript length: 3727 bps, Translation length: 505 residues

\section{ENST00000538521 (PDIA3 Novel)}

\begin{abstract}
TAGCGCTGTTC9CCGGGTGTGGCGCTGCTTCTTGCCGCGGC CCGCCTCGCCGCTGCCTCCGACGTGCTAGAACTCACGGACGAC AACTTCGAGAGTCGCATCTCCGACACGGGCTCTGCGGGCCTCA TGCTCGTCGAGTTCTTCGCCCCCTGTTCCATTCCTGCTGGATTT GAAGAGGAGAGTCTCCTCCAGCCTCATTGAAACTCTTTTTGTGG ATTTGATGACCTTATGGATATTGCCAAAGTTTAAGGCAG TGGATTTTAGTCCCAGCCTTGCAGCTTACACACACACC TGGTTGCTCCCCAGATTTCTGGAGATTCTCATTCAGTGT GGACACTGCAA...
\end{abstract}

Exons: 14, Coding exons: 13 Transcript length: 2099 bps Translation length: 485 residues

Fig. 1 NGS results from prostate cell line RNA samples. a Differences in DNA sequence for the PDIA3 normal transcript isoform and PDIA3 Novel transcript isoform. b PDIA3 and PDIA3N expres-

Expression of PDIA3N in LNCaP cells was higher compared to PDIA3 ( $<<0.0001$; Fig. 3 ).

A generated protein structure model for PDIA 3 and PDIA3N are shown in Fig. 4. The protein sequence for PDIA3N contains 485 amino acids. The first fragment of the $\mathrm{N}$-terminus differs in 56 amino acids (36 amino acid substitutions and 20 amino acid deletions) from PDIA3 (Fig. 4a). PDIA3N had a higher confidence (C-Score) than PDIA3 (Fig. 4b, I-TASSER). At the secondary structure level, PDIA3N lacks an $\alpha$-helix. Furthermore, PDIA3N contains a truncated thioredoxin site in the $\mathrm{N}$-terminus (CGH from WCGH, Fig. 4a, b). Twenty-two out of the 56 altered amino acids in the $\mathrm{N}$-terminus of PDIA3N were confirmed to affect the structure and function of the protein (PolyPhen-2 and PROVEAN, Supplementary File 3). Analysis of subcellular localization for PDIA3N and PDIA3 showed PDIA3N to be present in the cytosol while PDIA3 is present in the endoplasmatic reticulum (ER) (Supplementary File 4). Binding capacity for PDIA3 and PDIA3N to $1,25(\mathrm{OH})_{2} \mathrm{D}_{3}$ revealed a putative binding site in the $\mathrm{N}$-terminus with an estimated free energy of binding of $-9.29 \mathrm{kcal} / \mathrm{mol}$ (estimated inhibition constant, $\mathrm{Ki}=155 \mathrm{nM})$ and $-8.21 \mathrm{kcal} / \mathrm{mol}(\mathrm{Ki}=958 \mathrm{nM}$, respectively) (Supplementary File 5). b

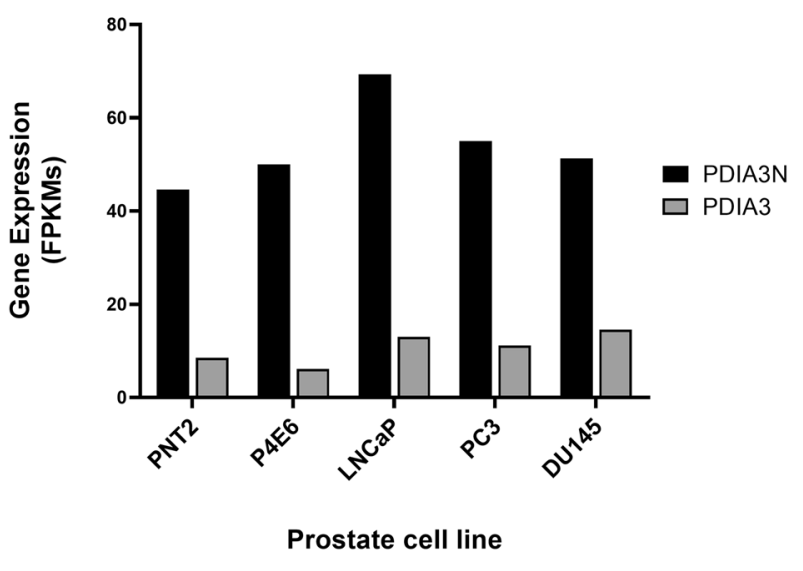

sion values (FPKM) obtained after cufflinks/2.1.1 software analysis of NGS results for prostate cell lines PNT2, P4E6, DU145, PC3 and LNCaP

\section{Discussion}

Isoforms of gene transcripts are suggested as plausible biomarkers for different diseases, such as cancer. The expression pattern of different isoforms may change depending on the stage or severity of disease and relation between expression of two gene isoforms can be correlated with a specific disease pattern $[9,34,35]$. Previous studies pinpoint PDIA3 as a plausible candidate for studying cancer prognosis as a target for cancer treatment $[18-21,36]$. The role of PDIA3 in cancer treatment remains controversial and implication of different PDIA3 transcript isoforms has not yet been studied in connection with progression of $\mathrm{PCa}$.

In the present study, a novel PDIA3 transcript isoform was detected in prostate cell lines, in line with previous studies on kidney and colon cells where PDIA3N is associated with cancer progression [24, 33]. Furthermore, PDIA3N has a higher mRNA expression compared to PDIA3 in prostate cells with especially high mRNA expression in LNCaP cells. Interestingly, this study indicates for a correlation between the ratio of PDIA3N/ PDIA3 and cancer stage. The structure of PDIA3N 

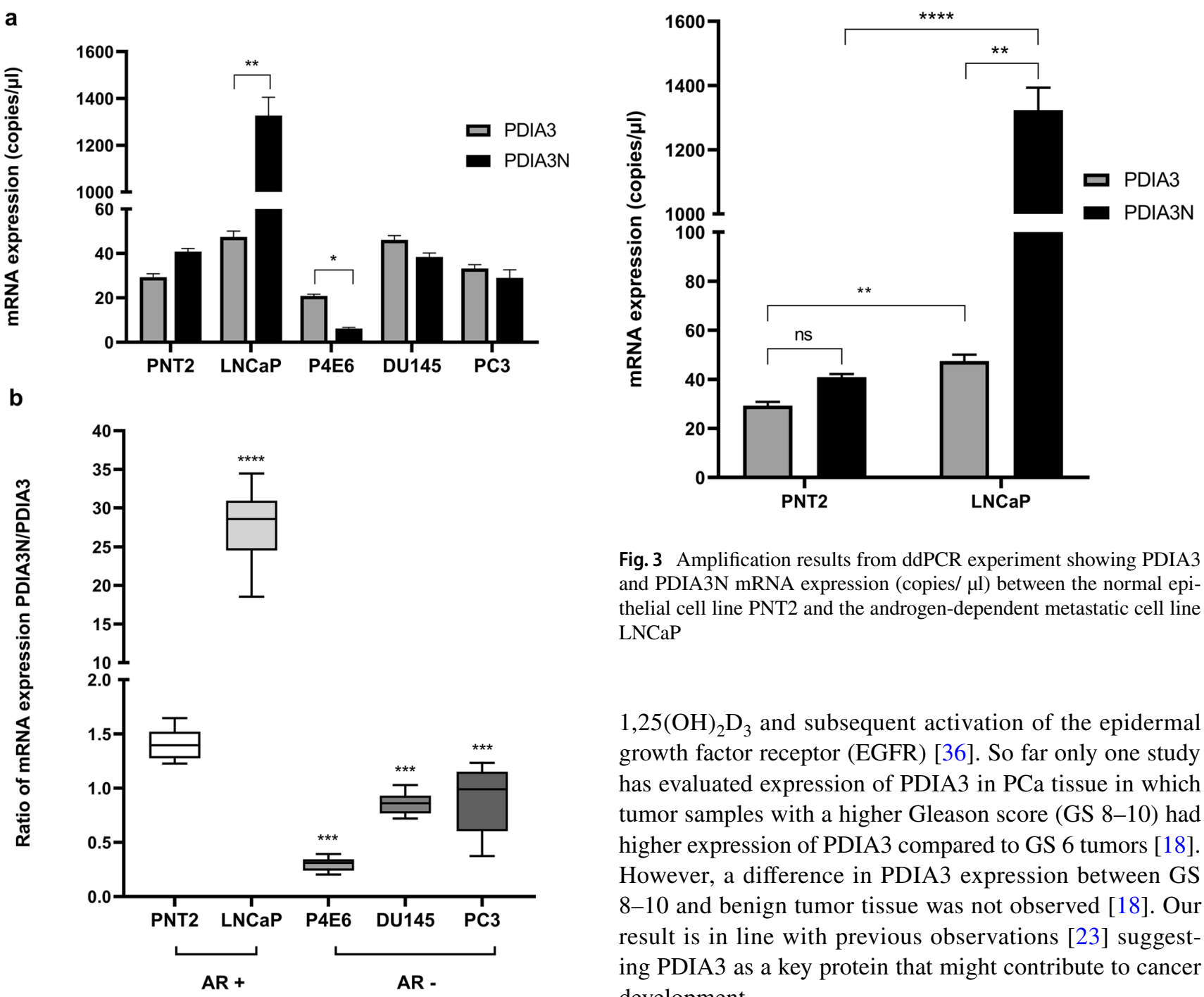

Fig. 2 Results from ddPCR absolute quantification experiments of cDNA samples from androgen receptor positive $(\mathrm{AR}+)$ cells, PNT2 and LNCaP; and androgen receptor negative (AR-) cells, P4E6, DU145 and PC3. a The mRNA expression (copies/ul) of PDIA3 and PDIA3N isoforms, and $\mathbf{b}$ the ratio of PDIA3N/PDIA3 mRNA expression

differed from PDIA3 suggesting an altered function of the protein concerning cell location, thioredoxin activity and affinity for $1,25(\mathrm{OH})_{2} \mathrm{D}_{3}$. Thus, a shift in ratio between PDIA3N and PDIA3 may contribute to cancer progression by decreased redox activity and altered hormone function. Furthermore, the ratio between PDIA3N and PDIA3 might be a relevant indicator to follow in future studies with focus on progression of PCa.

Several studies associate changed expression of PDIA3 with multiple pathologies including cancer and neurodegenerative disease [36-38]. Aberrant expression of PDIA3 is shown to be correlated with poor prognosis in several cancer types and increased cell proliferation mediated by

Fig. 3 Amplification results from ddPCR experiment showing PDIA3 and PDIA3N mRNA expression (copies/ $\mu \mathrm{l}$ ) between the normal epithelial cell line PNT2 and the androgen-dependent metastatic cell line $\mathrm{LNCaP}$

$1,25(\mathrm{OH})_{2} \mathrm{D}_{3}$ and subsequent activation of the epidermal growth factor receptor (EGFR) [36]. So far only one study has evaluated expression of PDIA3 in PCa tissue in which tumor samples with a higher Gleason score (GS 8-10) had higher expression of PDIA3 compared to GS 6 tumors [18]. However, a difference in PDIA3 expression between GS 8-10 and benign tumor tissue was not observed [18]. Our result is in line with previous observations [23] suggesting PDIA3 as a key protein that might contribute to cancer development.

An imbalance in ratio between VDR and PDIA3 (including PDIA3 isoforms) might contribute to PCa progression, as suggested in this study. An increased pool of PDIA3N would bind a large part of available $1,25(\mathrm{OH})_{2} \mathrm{D}_{3}$ and prevents, in part, the hormone to interact with VDR. However, VDR has a higher affinity to $1,25(\mathrm{OH})_{2} \mathrm{D}_{3}(\mathrm{Ki}$ of $1-10 \mathrm{nM})$ [15] compared to PDIA3 (Ki of $155 \mathrm{nM}$ ) and PDIA3N (Ki of $958 \mathrm{nM})$. This indicate that $1,25(\mathrm{OH})_{2} \mathrm{D}_{3}$ may preferentially bind to VDR but the larger pool of PDIA3 and PDIA3N will bind a considerable part of available $1,25(\mathrm{OH})_{2} \mathrm{D}_{3}$. Taken together, we suggest that the decreased availability of $1,25(\mathrm{OH})_{2} \mathrm{D}_{3}$ to VDR and lower levels of VDR have a negative impact on the antitumorigenic effects of $1,25(\mathrm{OH})_{2} \mathrm{D}_{3}$. The role of PDIA3N remains unclear but it may have a relevant role in pathogenesis due to its abundant expression in the metastatic androgen-dependent stage, compared to PDIA3, and due to its predicted aberrant structure and function. Thus, this study suggests that the different PDIA3 isoforms might play contrary roles in PCa progression. The switch in expression between these isoforms to a higher 
a

PDIA3, Lenght: 505 aa

MRLRRLALFPGVALLLAAARLAAASDVLELTDDNFES RISDTGSAGLMLVEFFAPWCGHCKRLAPEYEAAATRLKG IVPLAKVDCTANTNTCNKYGVSGYPTLKIFRDGEEAGAYDG PRTADGIVSHLKKQAGPASVPLRTEEEFKKFISDKDASIVGF FDDSFSEAHSEFLKAASNLRDNYRFAHTNVESLVNEYDDN GEGIILFRPSHLTNKFEDKTVAYTEQKMTSGKIKKFIQENIFG IC...

PDIA3N ,Length: 485 aa

MTLWILPKFKAVDFSPSLAAYTHTWLLPRFLEILIQCGH CKRLAPEYEAAATRLKGIVPLAKVDCTANTNTCNKYGVSGY PTLKIFRDGEEAGAYDGPRTADGIVSHLKKQAGPASVPLRT EEEFKKFISDKDASIVGFFDDSFSEAHSEFLKAASNLRDNY RFAHTNVESLVNEYDDNGEGIILFRPSHLTNKFEDKTVAYT EK...

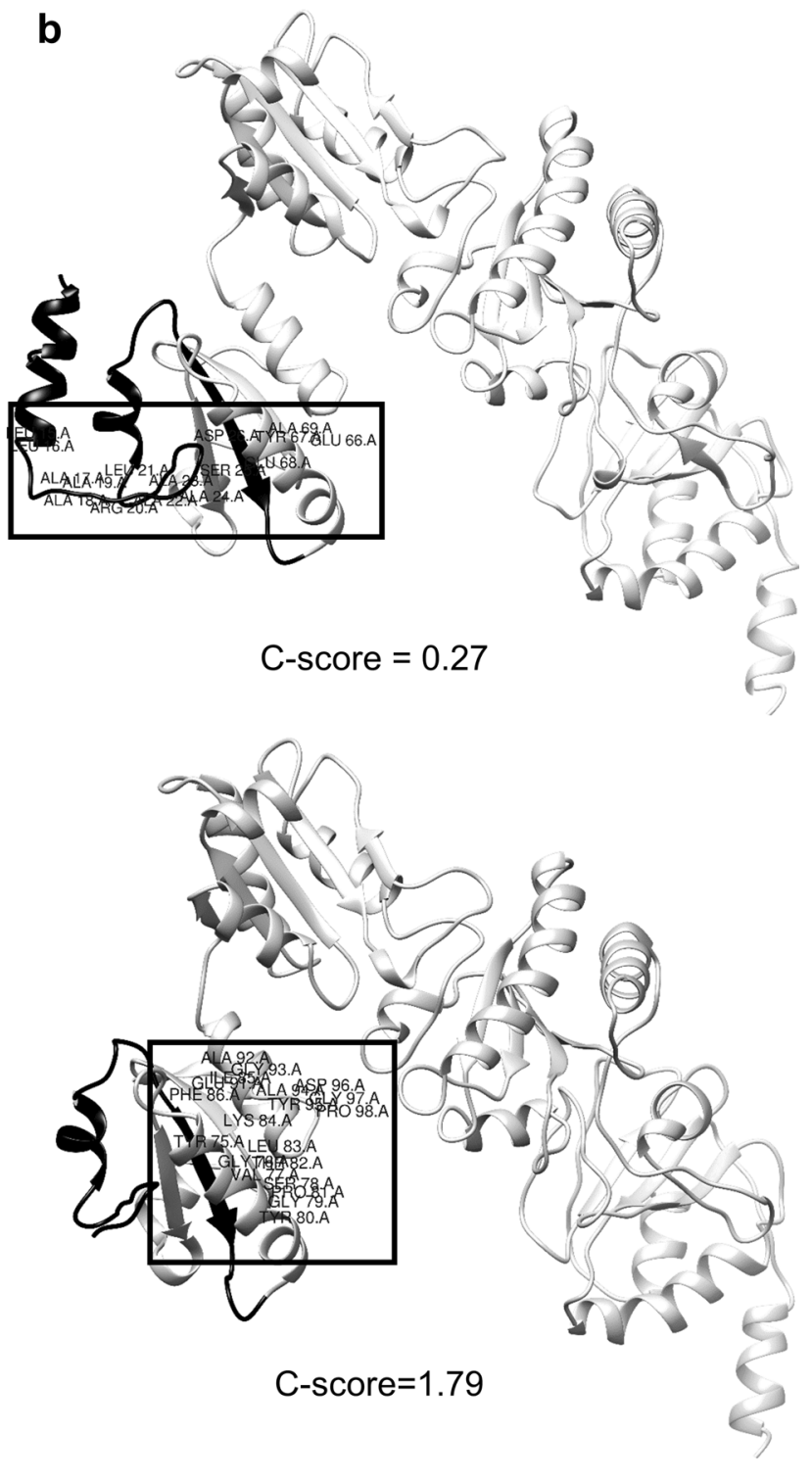

tein sequence that differs between both isoforms is highlighted in black and the amino acids that conform the $1,25(\mathrm{OH})_{2} \mathrm{D}_{3}$ binding pocket are labelled and marked with a rectangle in (b)

sequence in the $\mathrm{N}$-terminus and indicates that this isoform is mainly present in the cytosol, a different subcellular cell compartment compared to PDIA3 which in a major part is found in the ER and suggests a different function.

Normally, the major part of PDIA3 is associated with the ER, forming a complex with calreticulin and calnexin, participating in correct folding and in quality control of neosynthesized glycoproteins to be secreted or to be localized to the cell membrane [32, 41-43]. According to sequence analyses in the present study, a major part of the PDIA3 are present in the cytosolic compartment. Previous studies have demonstrated that PDIA3 forms complexes with the retinoic acid receptor [44], STAT3 [45-47], NF-אB [48] and 
Ref-1 [49] in the cytosol. Considering the thioredoxin sites and redox regulating capacity of PDIA3, it might impact the activity of complex bound proteins as well as regulating the redox potential within the cell. The predicted functional differences of PDIA3N compared to PDIA3, points out, according to our analyses, a cytosolic location. The structural change where one $\alpha$-helix is missing in PDIA3Ns secondary structure and its truncation in one active thioredoxin site indicate for an impaired thioredoxin activity and might be the cause of a lower binding affinity to $1,25(\mathrm{OH})_{2} \mathrm{D}_{3}$. The functional differences of PDIA3N might in turn lead to impaired functions of the complexes formed with PDIA3N in the cytosol.

Altogether, these data suggest that the novel transcript isoform of PDIA3, PDIA3N, is a putative candidate for studying progression of PCa. Future expression analyses of PDIA3 and PDIA3N in clinical samples, correlated to the Gleason score and androgen dependency stage of PCa, are necessary to determine if PDIA3N is a suitable biomarker for advanced PCa as well as the role of PDIA3, PDIA3N and the vitamin $\mathrm{D}$ endocrine system in regulating prostate cell biology.

Supplementary Information The online version contains supplementary material available at https://doi.org/10.1007/s11033-021-06277-1.

Acknowledgements We thank the students of the Biomedical Laboratory Science program at Jönköping University, Pontus Karlsson and Gustav Högberg, for their contribution in some of the experiments in this study.

Author's contributions DL Conceptualization, Data curation, Investigation, Methodology, Supervision, Writing-Reviewing and Editing; MA DC Investigation, Methodology, Data curation, Formal analysis, Validation, Visualization, Writing-Reviewing and Editing; DL Data curation, Investigation, Methodology, Software, Writing-Reviewing \& Editing; FS, SK and MF Data curation, Supervision, Writing-Reviewing and Editing.

Funding Open access funding provided by Jönköping University. This study was financially supported by Högskolans Jubileumsfond at the University College of Skövde (Dnr HS 2015/536). Jönköping University provided with the necessary resources to carry out this investigation.

\section{Declarations}

Conflicts of interest All authors declare that they have no conflicts of interest.

Open Access This article is licensed under a Creative Commons Attribution 4.0 International License, which permits use, sharing, adaptation, distribution and reproduction in any medium or format, as long as you give appropriate credit to the original author(s) and the source, provide a link to the Creative Commons licence, and indicate if changes were made. The images or other third party material in this article are included in the article's Creative Commons licence, unless indicated otherwise in a credit line to the material. If material is not included in the article's Creative Commons licence and your intended use is not permitted by statutory regulation or exceeds the permitted use, you will need to obtain permission directly from the copyright holder. To view a copy of this licence, visit http://creativecommons.org/licenses/by/4.0/.

\section{References}

1. Dong Z, Liu Y, Scott KF, Levin L, Gaitonde K, Bracken RB et al (2010) Secretory phospholipase A2-IIa is involved in prostate cancer progression and may potentially serve as a biomarker for prostate cancer. Carcinogenesis 31(11):1948-1955

2. Stangelberger A, Waldert M, Djavan B (2008) Prostate cancer in elderly men. Rev Urol 10(2):111-119

3. Hoang DT, Iczkowski KA, Kilari D, See W, Nevalainen MT (2017) Androgen receptor-dependent and -independent mechanisms driving prostate cancer progression: opportunities for therapeutic targeting from multiple angles. Oncotarget 8(2):3724-3745

4. Saini S (2016) PSA and beyond: alternative prostate cancer biomarkers. Cell Oncol (Dordr) 39(2):97-106

5. Farran B, Dyson G, Craig D, Dombkowski A, Beebe-Dimmer JL, Powell IJ et al (2018) A study of circulating microRNAs identifies a new potential biomarker panel to distinguish aggressive prostate cancer. Carcinogenesis 39(4):556-561

6. Trincado JL, Sebestyen E, Pages A, Eyras E (2016) The prognostic potential of alternative transcript isoforms across human tumors. Genome medicine 8(1):85

7. Albano F, Zagaria A, Anelli L, Coccaro N, Tota G, Brunetti C et al (2015) Absolute quantification of the pretreatment PMLRARA transcript defines the relapse risk in acute promyelocytic leukemia. Oncotarget 6(15):13269-13277

8. Zhu GQ, Zhou YJ, Qiu LX, Wang B, Yang Y, Liao WT et al (2019) Prognostic alternative mRNA splicing signature in hepatocellular carcinoma: a study based on large-scale sequencing data. Carcinogenesis. https://doi.org/10.1093/carcin/bgz073

9. Vitting-Seerup K, Sandelin A (2017) The landscape of isoform switches in human cancers. Mol Cancer Res 15(9):1206-1220

10. Feldman D, Krishnan AV, Swami S, Giovannucci E, Feldman BJ (2014) The role of vitamin D in reducing cancer risk and progression. Nat Rev Cancer 14(5):342-357

11. Petrou S, Mamais I, Lavranos G, I PT, Chrysostomou S. (2018) Effect of Vitamin D Supplementation in Prostate Cancer: A Systematic Review of Randomized Control Trials. Int J Vitamin Nutri Res Internationale Zeitschrift fur Vitamin- und Ernahrungsforschung 88(1-2):100-112

12. Zhu Y, Chen P, Gao Y, Ta N, Zhang Y, Cai J et al (2018) MEG3 Activated by vitamin D inhibits colorectal cancer cells proliferation and migration via regulating clusterin. EBioMedicine 30:148-157

13. Chen J, Lobachev KS, Grindel BJ, Farach-Carson MC, Hyzy SL, El-Baradie KB et al (2013) Chaperone properties of pdia3 participate in rapid membrane actions of 1alpha,25-dihydroxyvitamin d3. Molecular endocrinology (Baltimore, Md) 27(7):1065-1077

14. Boyan BD, Chen J, Schwartz Z (2012) Mechanism of Pdia3dependent 1alpha,25-dihydroxy vitamin D3 signaling in musculoskeletal cells. Steroids 77(10):892-896

15. Holmén J, Jansson A, Larsson D (2009) A kinetic overview of the receptors involved in 1,25-dihydroxyvitamin D3 and 24,25-dihydroxyvitamin D3 signaling: a systems biology approach. Crit Rev Eukaryot Gene Expr 19(3):181-196 
16. Cheng HS, Lee JXT, Wahli W, Tan NS (2019) Exploiting vulnerabilities of cancer by targeting nuclear receptors of stromal cells in tumor microenvironment. Mol Cancer 18(1):51

17. Campbell FC, Xu H, El-Tanani M, Crowe P, Bingham V (2010) The yin and yang of vitamin D receptor (VDR) signaling in neoplastic progression: operational networks and tissue-specific growth control. Biochem Pharmacol 79(1):1-9

18. Pressinotti NC, Klocker H, Schafer G, Luu VD, Ruschhaupt M, Kuner R et al (2009) Differential expression of apoptotic genes PDIA3 and MAP3K5 distinguishes between low- and high-risk prostate cancer. Mol Cancer 8:130

19. Zhao G, Lu H, Li C (2015) Proapoptotic activities of protein disulfide isomerase (PDI) and PDIA3 protein, a role of the Bcl-2 protein Bak. J Biol Chem 290(14):8949-8963

20. Takata H, Kudo M, Yamamoto T, Ueda J, Ishino K, Peng WX et al (2016) Increased expression of PDIA3 and its association with cancer cell proliferation and poor prognosis in hepatocellular carcinoma. Oncol Lett 12(6):4896-4904

21. Ye Q, Fu P, Dou J, Wang N (2018) Downregulation of PDIA3 inhibits proliferation and invasion of human acute myeloid leukemia cells. Onco Targets Ther 11:2925-2935

22. Uhlen M, Zhang C, Lee S, Sjostedt E, Fagerberg L, Bidkhori G, et al. A pathology atlas of the human cancer transcriptome. Science (New York, NY). 2017;357(6352).

23. Untergasser A, Nijveen H, Rao X, Bisseling T, Geurts R, Leunissen JA. Primer3Plus, an enhanced web interface to Primer3. Nucleic acids research. 2007;35(Web Server issue):W71-4.

24. UniProt Consortium T (2018) UniProt: the universal protein knowledgebase. Nucleic Acids Res 46(5):2699

25. Zhang Y (2008) I-TASSER server for protein 3D structure prediction. BMC Bioinformatics 9:40

26. Pettersen EF, Goddard TD, Huang CC, Couch GS, Greenblatt DM, Meng EC et al (2004) UCSF Chimera-a visualization system for exploratory research and analysis. J Comput Chem 25(13):1605-1612

27. Adzhubei IA, Schmidt S, Peshkin L, Ramensky VE, Gerasimova A, Bork P et al (2010) A method and server for predicting damaging missense mutations. Nat Methods 7(4):248-249

28. Choi Y, Sims GE, Murphy S, Miller JR, Chan AP (2012) Predicting the functional effect of amino acid substitutions and indels. PLoS ONE 7(10):e46688

29. Almagro Armenteros JJ, Sonderby CK, Sonderby SK, Nielsen H, Winther O (2017) DeepLoc: prediction of protein subcellular localization using deep learning. Bioinformatics (Oxford, England) 33(21):3387-3395

30. Morris GM, Huey R, Lindstrom W, Sanner MF, Belew RK, Goodsell DS et al (2009) AutoDock4 and AutoDockTools4: Automated docking with selective receptor flexibility. J Comput Chem 30(16):2785-2791

31. Rochel N, Wurtz JM, Mitschler A, Klaholz B, Moras D (2000) The crystal structure of the nuclear receptor for vitamin $\mathrm{D}$ bound to its natural ligand. Mol Cell 5(1):173-179

32. Gaucci E, Raimondo D, Grillo C, Cervoni L, Altieri F, Nittari $\mathrm{G}$ et al (2016) Analysis of the interaction of calcitriol with the disulfide isomerase ERp57. Sci Rep 6:37957

33. Zerbino DR, Achuthan P, Akanni W, Amode MR, Barrell D, Bhai J et al (2018) Ensembl 2018. Nucleic Acids Res 46(D1):D754-D761

34. Adamopoulos PG, Mavrogiannis AV, Kontos CK, Scorilas A (2019) Novel alternative splice variants of the human protein arginine methyltransferase 1 (PRMT1) gene, discovered using next-generation sequencing. Gene 699:135-144

35. Climente-Gonzalez H, Porta-Pardo E, Godzik A, Eyras E (2017) The Functional impact of alternative splicing in cancer. Cell Rep 20(9):2215-2226

36. Hettinghouse A, Liu R, Liu CJ (2018) Multifunctional molecule ERp57: From cancer to neurodegenerative diseases. Pharmacol Ther 181:34-48

37. Zhu Y, Cai L, Guo J, Chen N, Yi X, Zhao Y et al (2016) Depletion of Dicer promotes epithelial ovarian cancer progression by elevating PDIA3 expression. Tumour Biol 37(10):14009-14023

38. Trivedi R, Muller GA, Rathore MS, Mishra DP, Dihazi H (2016) Anti-leukemic activity of shikonin: role of ERP57 in Shikonin induced apoptosis in acute myeloid leukemia. Cell Physiol Biochem 39(2):604-616

39. Brar GA, Weissman JS (2015) Ribosome profiling reveals the what, when, where and how of protein synthesis. Nat Rev Mol Cell Biol 16(11):651-664

40. Kochetov AV (2008) Alternative translation start sites and hidden coding potential of eukaryotic mRNAs. Bio Essays 30(7):683-691

41. Oliver JD, van der Wal FJ, Bulleid NJ, High S (1997) Interaction of the thiol-dependent reductase ERp57 with nascent glycoproteins. Science (New York, NY) 275(5296):86-88

42. Molinari M, Helenius A (1999) Glycoproteins form mixed disulphides with oxidoreductases during folding in living cells. Nature 402(6757):90-93

43. Oliver JD, Roderick HL, Llewellyn DH, High S (1999) ERp57 functions as a subunit of specific complexes formed with the ER lectins calreticulin and calnexin. Mol Biol Cell 10(8):2573-2582

44. Zhu L, Santos NC, Kim KH (2010) Disulfide isomerase glucoseregulated protein 58 is required for the nuclear localization and degradation of retinoic acid receptor alpha. Reproduction (Cambridge, England) 139(4):717-731

45. Ndubuisi MI, Guo GG, Fried VA, Etlinger JD, Sehgal PB (1999) Cellular physiology of STAT3: Where's the cytoplasmic monomer? J Biol Chem 274(36):25499-25509

46. Chichiarelli S, Gaucci E, Ferraro A, Grillo C, Altieri F, Cocchiola $R$ et al (2010) Role of ERp57 in the signaling and transcriptional activity of STAT3 in a melanoma cell line. Arch Biochem Biophys 494(2):178-183

47. Guo GG, Patel K, Kumar V, Shah M, Fried VA, Etlinger JD et al (2002) Association of the chaperone glucose-regulated protein 58 (GRP58/ER-60/ERp57) with Stat3 in cytosol and plasma membrane complexes. J Interferon \& cytokine Res 22(5):555-563

48. Wu W, Beilhartz G, Roy Y, Richard CL, Curtin M, Brown L et al (2010) Nuclear translocation of the 1,25D3-MARRS (membrane associated rapid response to steroids) receptor protein and NFkappaB in differentiating NB4 leukemia cells. Exp Cell Res 316(7): 1101-1108

49. Grillo C, D’Ambrosio C, Scaloni A, Maceroni M, Merluzzi S, Turano C et al (2006) Cooperative activity of Ref-1/APE and ERp57 in reductive activation of transcription factors. Free Radical Biol Med 41(7):1113-1123

Publisher's Note Springer Nature remains neutral with regard to jurisdictional claims in published maps and institutional affiliations. 